\title{
Optimized Route Capability (ORC) Intelligent Offloading of Congested Arrival Routes
}

\author{
Shannon Zelinski* and Min Xue ${ }^{\dagger}$ \\ NASA Ames Research Center, Moffett Field, CA, 94035 \\ Philip Bassett $\ddagger$ \\ FAA Headquarters, Washington, D.C., 20591
}

\begin{abstract}
The Optimized Route Capability (ORC) concept is designed to enable intelligent offloading of congested arrival routes. When ORC predicts arrival route congestion as projected excess arrival meter fix delay, automation offers decision support to traffic managers by identifying candidate flights to strategically reroute to alternate meter fixes and alleviate the congestion. This concept was applied to a model of arrival operations into Houston International Airport. An arrival rush from the Northeast was simulated in fast-time to analyze ORC algorithm behavior. The results demonstrate how strategically rerouting a few flights to alternate meter fixes not only has the potential to manage meter fix delay (and possibly the need for traffic management initiatives applied upstream), but may also increase airport capacity utilization and reduce total flight delay.
\end{abstract}

\section{Introduction}

Traffic Management Units (TMUs) found in US Air Route Traffic Control Centers (ARTCCs) monitor flight traffic, weather patterns, and airspace and route status, identify potential problems to traffic flow, and respond with strategic and tactical adjustments to the flow. TMU responsibilities include very complex and time-intensive interactions with multiple facilities, area supervisors, and the control room floor. Due to constantly changing weather and traffic demand, there is often a small window of opportunity in which adjustments can be implemented without causing unforeseen complications. Adjustments made farther upstream are often preferable, resulting in the least impact on flight time and distance. However, the current practice tends toward postponing the adjustment decision and letting the scenario play out until the potential problem is more certain due to time-consuming verbal coordination required across facilities. The FAA's Optimized Route Capability (ORC) concept is envisioned to assist TMUs by conducting much of the laborious data gathering and interpretation necessary to identify, evaluate, and respond to changing conditions. ${ }^{1,2}$ The ORC concept is envisioned to interpret and integrate disparate data sources and consider multiple objectives (e.g. weather avoidance, competing flow demands, workload considerations) when generating optimal airspace and routing configurations that allow for timely and seamless coordination beyond the immediate and adjoining control facilities. This concept requires ORC to provide reroute recommendations for individual flights when projected demand exceeds capacity in defined areas of the National Airspace System (NAS). ORC is also required to take into account the effect of its recommendations on metrics such as extra track distance, delay, and resource loading that are currently either unavailable or too difficult for a traffic management coordinator to discern. This data-driven approach to formulating routing solutions is envisioned to facilitate cross facility coordination, allowing for timely and seamless implementation of changes.

The original research assessment identified Metroplex environments, where there are many airport interactions, high traffic volume, and complex resource-utilization challenges, as particularly well-suited for

\footnotetext{
*Research Engineer, Aerospace High Density Operations Branch. Mail Stop 210-6. AIAA Senior Member.

${ }^{\dagger}$ Research Scientist, Systems Modeling \& Optimization Branch. Mail Stop 210-8. AIAA Senior Member

${ }^{\ddagger}$ FAA NextGen ORC Program Manager, NextGen Concept Development \& Validation Branch
} 
the ORC concept. ${ }^{1}$ The concept not only provides automation support to identify alternate arrival and departure routes to underutilized resources, but to dynamically utilize airspace nominally reserved for segregating arrivals and departures or flows to or from other airports. NASA has recently teamed with the FAA to facilitate initial algorithm development. This initial phase of ORC focuses on intelligent offloading of congested arrival routes into high density airports. ${ }^{3}$ High demand at a few arrival meter fixes can cause unacceptable arrival scheduling delay to be passed from terminal to en-route airspace, while other arrival meter fixes to the same Terminal Radar Approach Control (TRACON) have available capacity. ORC will monitor projected arrival scheduling delay at each arrival meter fix. If excessive delay is predicted, ORC evaluates the effects of available reroute combinations from the over-utilized arrival meter fix to those that are less utilized, and selects a relatively low-cost set of individual flight reroutes to recommend to the TMU for implementation.

This paper describes the ORC concept for intelligent offloading of congested arrival routes (hereafter referred to simply as ORC). The initial ORC algorithm and it's implementation in a fast-time simulation model of Houston International Airport (IAH) arrival operations is presented. A fast-time simulation of an aggressive arrival rush into IAH's Northeast corner post meter fix is conducted to exercise and stress the algorithm and provide proof-of-concept. An analysis of ORC algorithm behavior is presented along with a discussion of possible directions for algorithm improvement.

\section{ORC Intelligent Offloading Concept}

Offloading congested arrival routes is a function currently performed for only airborne flights, by necessity, and in a reactive manner. When it is apparent to a traffic management coordinator (TMC) in the ARTCC TMU that a group of airborne flights will overload an arrival fix to an extent that en-route vectoring to deliver a manageable feed to terminal arrival controllers will become unmanageable, the TMC selects flights to reroute to other under-utilized arrival fixes. However, the problem is usually recognized so late that only the last few flights in the group can be rerouted en-route, leaving the remainder of the problem to be handled tactically as transition airspace reroutes or TRACON vectors. This not only utilizes less efficient trajectory options, but puts additional strain on the already overloaded en-route arrival sectors and TRACON. Predeparture aircraft are not generally considered for reroutes to alternate meter fixes. Rather, they are delayed on the ground until a slot opens up in the congested arrival stream, which may take far longer than rerouting to an alternate meter fix with acceptable additional fuel burn.

Figure 1 depicts a notional representation of offloading congested arrival routes. Four orange dots depict arrival meter fixes. Blue lines feeding into the meter fixes are Standard Terminal Arrival Routes (STARs).

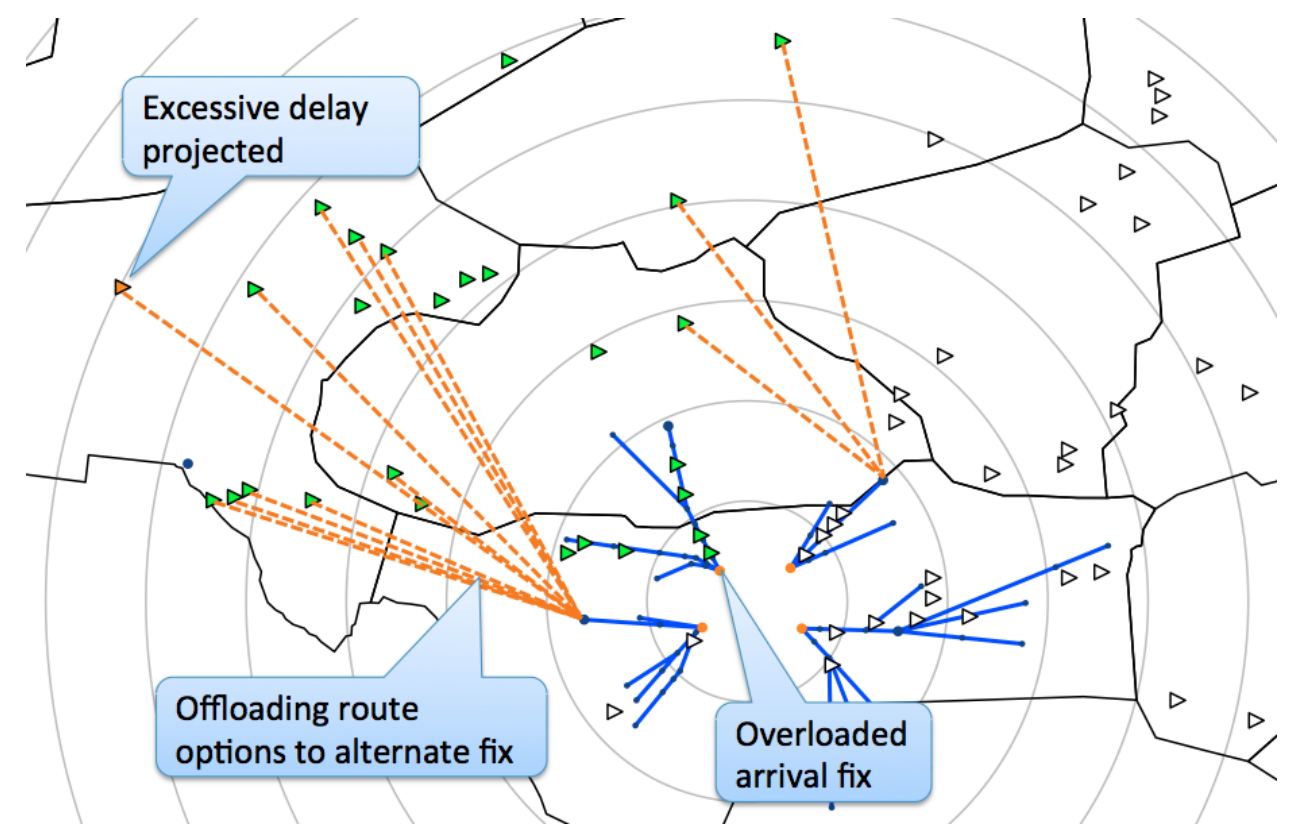

Figure 1. Offloading congested arrival routes to alternate meter fixes 
Triangles represent arrival flights. Due to the abundance of flights in the Northwest, the orange highlighted flight is projected to have excessive meter fix delay indicating meter fix overload. Flights originally bound for the overloaded meter fix are highlighted in green. Several have the option to reroute to an alternate meter fix, shown as dashed orange lines to STAR transitions to neighboring meter fixes. After analyzing route efficiency and scheduling costs associated with these available reroute options, ORC selects a subset of these reroutes to display in the TMU as suggested reroutes to alleviate congestion.

One of the challenges associated with the earlier ORC concept was a lack of reliable capacity estimation capabilities to identify problems. ${ }^{4}$ Flow capacity has typically been managed by placing a miles-in-trail (MIT) restriction at a metering point along the flow. But this method of defining flow capacity requires an operations expert to periodically evaluate the situation and adjust the capacity. This often results in inaccurate or excessive Traffic Management Initiatives. ORC's new focus on arrival route congestion enables it to adopt a TBFM approach to capacity driven by arrival scheduling. ${ }^{\text {a }}$ ORC monitors TBFM arrival schedules 90 minutes in advance, leaving ample opportunity for strategic reroutes with less impact to flight time and distance. Because of the uncertainty associated with estimating arrival schedules 90 minutes in advance, a user-defined threshold for projected meter fix delay is used to predict arrival route congestion. Even when arrival scheduling is not being used as intended for terminal metering, TMCs use large meter fix delay estimates as indicators of arrival route congestion requiring their attention. IAH TMCs identified seven minutes as the amount of delay seen on the TBFM time-line they associate with the need for rerouting or passing flow restrictions upstream via Traffic Management Initiatives. When ORC predicts an arrival fix overload (flight meter fix delay exceeding seven minutes for IAH), ORC reroute options for all flights bound for the overloaded fix (including pre-departure aircraft within the 90-minute planning horizon) are filtered to determine reroute availability for each individual flight. All possible combinations of available individual flight reroutes are then prioritized and evaluated to select a solution that provides acceptable arrival fix delay with minimal impact to flight operators. ORC then displays the suggested set of individual flight reroutes to TMCs along with the cost metrics used to select them to help TMCs determine whether to implement the reroutes as suggested, modify them and recalculate their estimated effects, or ignore them.

\section{ORC Automation Functions}

The ORC concept relies on the integration of its own decision support automation with other automation such as the FAA's Traffic Flow Management System (TFMS) for trajectory and meter fix arrival time prediction and Time Based Flow Management (TBFM) for estimates of arrival scheduling delay pushed into en-route airspace. The concept is also dependent on the human TMC's ability to quickly evaluate and implement recommended individual flight reroutes. Figure 2 shows the control loop where ORC decision support automation functions (blue) interact with external automation functions (orange) and the TMC. The main focus of this paper is on the ORC decision support automation. Whereas envisioned inputs and outputs to external human and automation systems are discussed, the manner in which they interface is not discussed in detail.

As shown in Fig. 2, ORC automation functions are broken down into four basic functions discussed in detail in the following subsections. These functions are repeated periodically as flight states and constraints are updated. Predict Arrival Route Congestion monitors estimated flight delay at the meter fix from the TBFM arrival scheduler. ORC continues to monitor delay through each update cycle until arrival route congestion is predicted. Determine Reroute Availability filters all flights to just those that currently have a feasible reroute to another meter fix available. Evaluate Reroute Combinations prioritizes the list of available reroutes and probes the Trajectory Predictor and Arrival Scheduler functions to generate evaluation metrics for combinations of available reroutes. Finally, when a set of reroutes is found for which the evaluation metrics satisfy solution criteria, Recommend Reroute Solution displays the reroute solution to the TMC. The TMC may modify the solution (or come up with a completely different solution) and instruct the decision support automation to re-evaluate to see the effect of the new solution on evaluation metrics. It is envisioned that the chosen ORC solution will be displayed on the TFMS system for expedited coordination and delivery of the information. From the ORC algorithm perspective, reroutes take effect when inputs to Predict Arrival Route Congestion from the external Trajectory Predictor and Arrival Scheduler reflect the change in flight plans.

a TBFM arrival scheduling is also known as terminal metering among air traffic operations experts. 


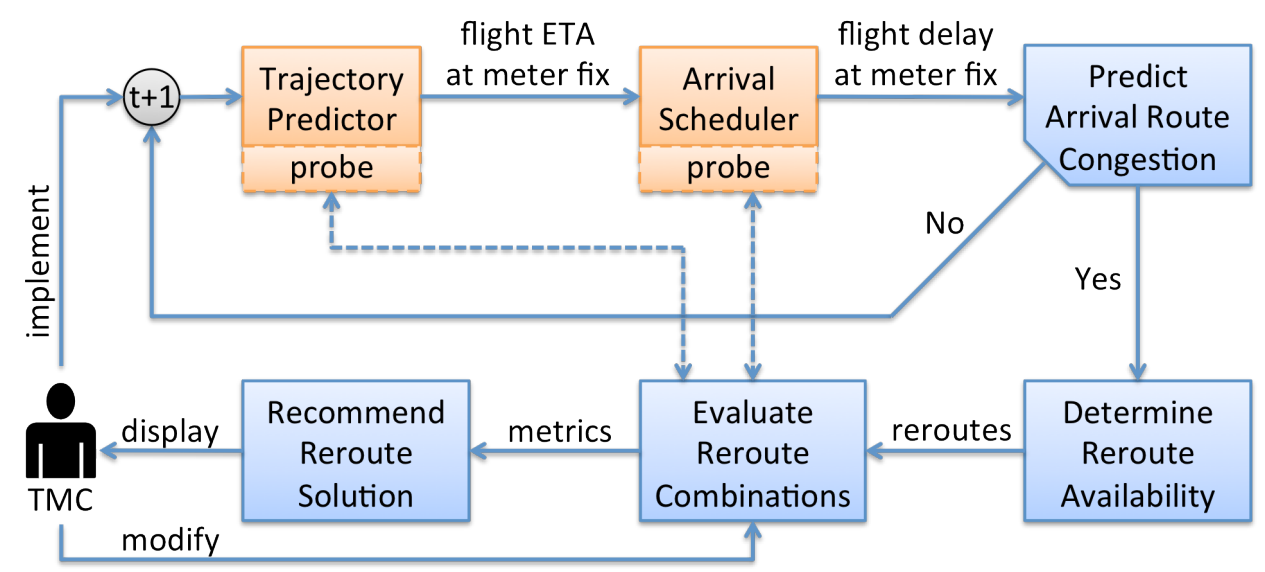

Figure 2. ORC automation functions

\section{III.A. Predict Arrival Route Congestion}

ORC monitors arrival scheduling meter fix delay to predict arrival route congestion. Arrival scheduling uses flight estimated time of arrival (ETA) at the meter fix along with detailed models of nominal TRACON routing, spacing requirements, delay buffers, and runway assignment logic to assign scheduled times of arrival (STA) at the runway and meter fix for each flight. Delay at the arrival meter fix (meter fix STA minus ETA) is first estimated for a flight when the flight plan is processed by the Trajectory Predictor to generate a meter fix ETA. Meter fix delay estimates are then updated every minute along with the flight trajectory predictions. For all flights with runway ETAs within the 90-minute planning horizon, if an individual flight delay $d_{i}$ at it's meter fix exceeds the user defined detection threshold $D$ (set to 7 minutes for IAH), arrival route congestion is predicted and ORC proceeds to the Determine Route Availability function.

\section{III.B. Determine Reroute Availability}

All ORC reroutes are direct route segments from a flight's current position to transition fixes for existing STARs, streamlining the process of rerouteing to the alternate meter fix. However, not all direct routes from current position to STARs may be feasible. Therefore filters are used to generate a subset of available individual reroute options. At a minimum, meter fix and geographical filters are used to limit the available reroute options. The meter fix filter ensures that all flights eligible for ORC reroutes are currently assigned to a meter fix for which arrival route congestion has been predicted. This saves ORC the trouble of evaluating reroutes that do not offload the source of the predicted problem.

The geographical filters attempt to address common operational constraints for reroutes with generic rules related to published adaptation data. Two different geographical filters were considered. First, flights were required to be within a +/-90 degree capture angle from the arrival transition leading to the alternate meter fix. This ensures that if a flight flew direct to the transition fix, it would need to turn no more than 90 degrees to join the STAR transition path once reaching the transition fix. Second, flights may not be within 5 minutes of crossing a Center boundary when rerouting. Operations experts insisted that they would not reroute a flight so close to hand-off between Centers due to the additional coordination required. This was enforced by filtering flights from reroute consideration if their projected dead reckoning trajectory (based on current position, heading, and speed) crossed a Center boundary within 5 minutes.

Figure 3 illustrates the geographical filters applied to IAH. The orange dots are meter fixes and the blue lines are STAR transitions to their respective meter fixes. A sample transition fix used to enter the STAR is labeled for each meter fix. All flights shown in this example are approaching the Northeast meter fix assumed to be overloaded and in need of offloading. Flight 3 is projected to cross a Center boundary within 5 minutes and so it is filtered from reroute consideration. Flights 1 and 2 are not projected to cross a Center boundary within 5 minutes. The arrival procedure capture angle filter determines which reroute options are available to them. Blue shaded regions highlight +-90 degree capture angle ranges around candidate arrival transitions beginning at SAT, CVE, SWB, and JEPEG. Even though the flights are within capture angle range of SWB, it leads to the same overloaded meter fix to which the flights are already headed, so SWB is 
not a reroute option. Flight 1 is within capture angle range of CVE and so this reroute option is highlighted in yellow. Flight 2 is within capture angle range of both CVE and JEPEG and so both these reroute options are highlighted in yellow.

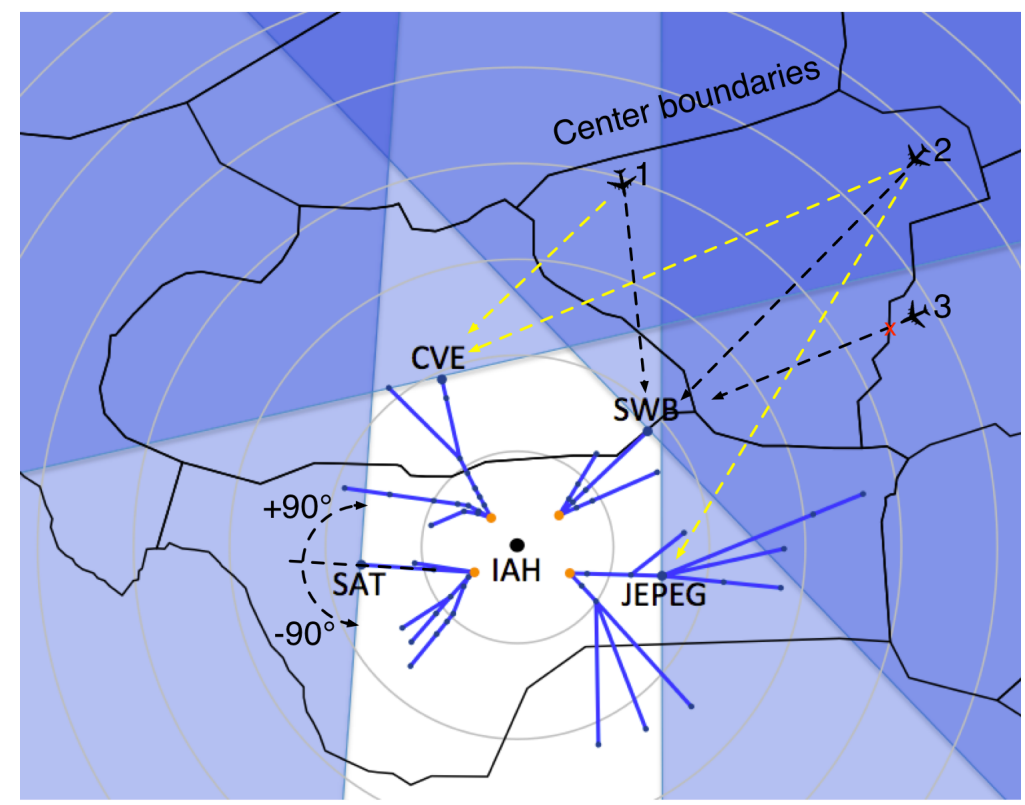

Figure 3. Geographical reroute filters

Based on discussions with operations experts, TMCs are hesitant to reroute international flights due to crew and fuel constraints. Therefore, an international flight filter removed from reroute consideration all flights with a 4-letter origin airport code not beginning with "K". Additionally, flights that had already been rerouted previously by ORC, were filtered from reroute consideration.

\section{III.C. Evaluate Reroute Combinations}

Given a set of available reroute options, Evaluate Reroute Combinations calculates cost metrics by probing the external trajectory prediction and arrival scheduling functions with various reroute options. Reroute cost metrics only require a single probe of the trajectory predictor per reroute, whereas scheduling cost metrics require probing the arrival scheduler for every possible combination of reroutes, which is much more computationally intensive. Therefore, reroute cost metrics are used to prioritize the set of combinations to hasten finding a suitable solution. ORC considers a single reroute cost metric, flight time difference, measured as the change in runway ETA between the current route and the reroute, based on nominal speed profile.

ORC considered a single scheduling cost metric which was excess meter fix delay $\left(d_{i}-D\right.$, where $D=7$ minutes). Any reroute combination for which all flights had a scheduling cost less than or equal to zero was considered to be a complete solution. However, if no complete solutions existed, a scheduling cost function was used in the following Recommend Reroute Solution process to identify the best partial solution.

Figure 4 shows the Evaluate Reroute Combinations process. The process begins by calculating reroute cost metrics for the set of available reroutes from the Determine Reroute Availability function. The reroutes are sent to the trajectory predictor to calculate meter fix ETAs, which are extended to runway ETAs using nominal TRACON transit time based on the same adaptation used by the arrival scheduler. The reroute runway ETA is compared with the original runway ETA to calculate the flight time difference. The cost of a combination of reroutes is simply the sum of the respective individual reroute costs.

Next, all possible combinations of reroutes are sorted by reroute cost into a prioritized list. Scheduling costs are computed for each combination of reroutes in order of priority by probing the arrival scheduler with meter fix and ETA updates to the rerouted flights and comparing $d_{i}$ with $D$. Evaluate Reroute Combinations ends when either a combination is found to be a complete solution $\left(d_{i}-D<=0\right.$ for all flights), or the entire prioritized list has been evaluated. Cost metrics for all evaluated combinations are stored for consideration in the Recommend Reroute Solution function. 


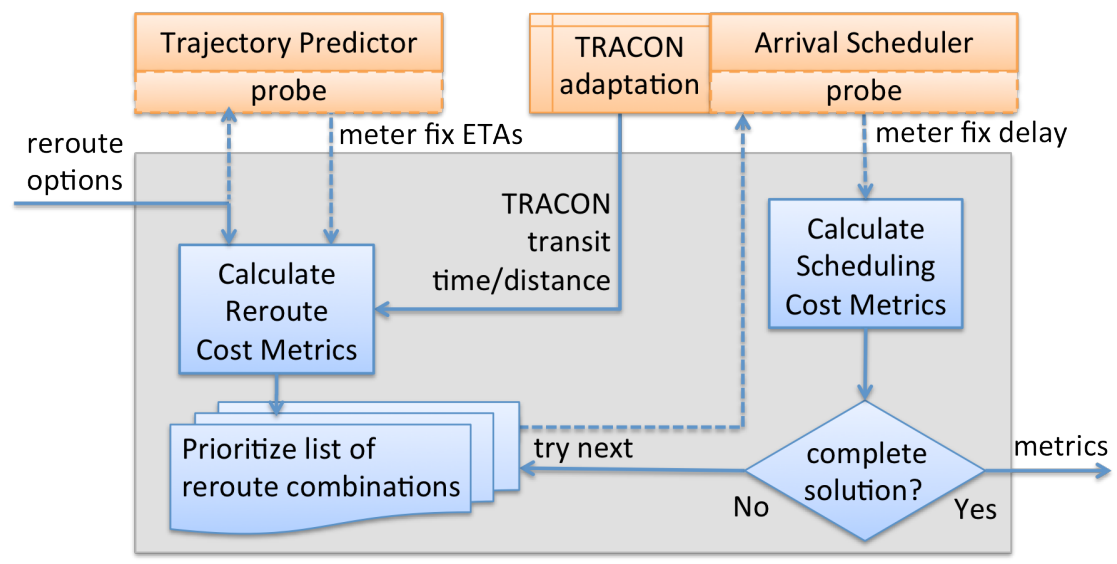

Figure 4. Evaluate Reroute Combinations process

\section{III.D. Recommend Reroute Solution}

The final ORC automation function selects a reroute combination to recommend to the TMC. A scheduling cost function was designed to select a complete solution should one exist, but otherwise select a partial solution that reduced the scheduling cost as uniformly as possible among flights. The objective of the scheduling cost function was to minimize the maximum excess meter fix delay for an individual flight. For all complete solutions, the maximum excess meter fix delay is less than or equal to zero. All other reroute combinations constituting a partial solution will have a value greater than zero; however the minimum partial solution ensures than no single flight is left with larger excess delay than necessary. The outcome of this reroute solution selection process is that reroute cost is only considered (via candidate solution prioritization) in so far as a complete solution can be found and all combinations of lower priority (higher reroute cost) are not evaluated. If no complete solution is found and the entire prioritized list is evaluated, partial solution selection is driven by scheduling cost alone. The assumption is that the systemic goal of reducing maximum excess delay is more important than individual flight efficiency.

\section{Simulation Implementation}

For the purposes of demonstrating a proof-of-concept, ORC automation was implemented in a fasttime simulation of IAH arrivals. Models of TFMS trajectory prediction and TBFM arrival scheduling were integrated with ORC automation coded in the Python scripting language to complete the simulation.

\section{IV.A. Trajectory Prediction}

The Future ATM Concepts Evaluation Tool (FACET) ${ }^{5}$ was used to model the Trajectory Prediction function. FACET is a simulation software tool developed by NASA. FACET can quickly generate and simulate thousands of aircraft trajectories using aircraft performance profiles, airspace models, weather data, and flight schedules and flight plans. It models trajectories for the climb, cruise, and descent phases of flight for each type of aircraft using the Base of Aircraft Data (BADA) database provided by Eurocontrol. ${ }^{6}$ FACET provides researchers with a simulation environment for preliminary testing of advanced ATM concepts through its Application Programming Interface (API).

Given a traffic scenario of flight plans, FACET was used to simulate all flights along unimpeded trajectories using nominal-speed profiles and historic wind data. In order to make use of FACET's capability of trajectory prediction, alternate flight plans or routes via different IAH arrival meter fixes were generated according to the published STARs, and these flight plans were fed into FACET for generating unimpeded trajectories without conflict detection and resolution. The ETAs at alternative meter fixes were then retrieved and used as inputs to the arrival scheduler described in following section. To include the wind effect, the Rapid Refresh (RAP) data provided by National Oceanic \& Atmospheric Administration (NOAA) were used as wind data resource, which is reloaded hourly in FACET. As FACET uses the nominal airspeed for a given aircraft type when generating trajectories, speed restrictions in STARs were imposed additionally in 
FACET via API script.

Runway ETAs were estimated by adding to the meter fix ETAs the nominal zero-wind transit time from meter fixes to their primary runways according to TBFM adaptation.

\section{IV.B. Arrival Scheduling}

Researchers consulted with IAH Center and TRACON controllers and TBFM experts to understand desired characteristics of arrival flows transitioning from Center to Terminal airspace. As both the triggering and evaluating mechanism for ORC, it is extremely important for the Arrival Scheduling function to provide a scheduled arrival flow that is desirable for both Center and TRACON controllers. A simplified model of the TBFM arrival scheduling currently fielded at IAH was developed to reduce computation time for iterative use. Consultation with Houston subject matter experts ensured the simplified algorithm retained key operational considerations.

Arrivals enter Houston TRACON (I90) via four arrival gates positioned in the northwest, northeast, southwest, and southeast corners. IAH arrivals enter I90 via six main meter fixes, one for each Southern gate and two for each Northern gate. Figure 5 shows the STAR transition routes to the Northern meter fixes feeding IAH. The transitions to the more Northern meter fixes MPORT and WHACK are the preferred arrival routes. The dual meter fixes at each of the Northern gates are often treated as a single stream due to the complexity of blending two streams when there is a merge shortly after entering the TRACON or runway dependencies. This is known as mirrored metering. In the future, ORC may be used to balance flows to the same arrival gate by modeling TRACON merge point scheduling constraints, but this proof-of-concept focuses on offloading to different arrival gates. Therefore, traffic scenarios were generated such that all flights filed to the preferred MPORT and WHACK meter fixes. As seen in Fig. 5, only one transition fix BRKAT does not have a published STAR transition to the preferred meter fix. BRKAT is rarely if ever used in practice and so traffic scenarios did not include flights using this transition.

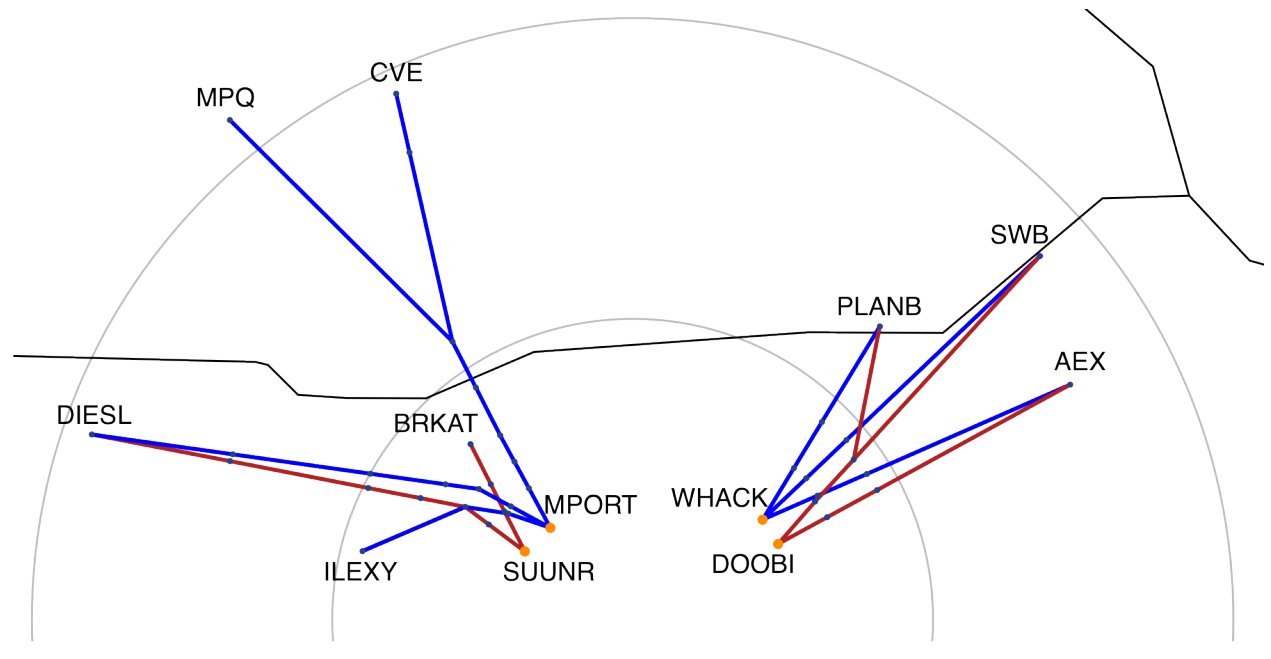

Figure 5. Published STAR transitions to North meter fixes feeding IAH.

On a first-come-first-served basis the scheduler computes the earliest feasible schedule times for a flight at the meter fix and runway subject to their respective simplified separation constraints and allowable transit time ranges between them. ${ }^{7}$

\section{IV.B.1. Separation Constraints}

I90 TBFM nominal separation constraints at the meter fixes are set to $6 \mathrm{NM}$ with the exception of the northwest meter fixes, which are set to $7 \mathrm{NM}$. This provides a 1-2 NM buffer over the $5 \mathrm{NM}$ minimum to account for compression. The winds aloft in Houston airspace are predominantly from the West, often subjecting flows from the West to strong tailwinds, and flows from the East to strong headwinds. Experienced Center controllers feeding the northeast corner can deliver to the northeast meter fixes with as little as 5.5 NM separation to accommodate high demand. Whereas Center controllers feeding the northwest corner 
insist that higher ground speeds due to westerly winds require them to deliver to the northwest meter fixes with 8-10 NM separation.

I90 terminal airspace controllers expressed concern at how the increased use of optimized profile descents (OPD) has not only limited their use of lateral delay maneuvers on these flights but has reduced the nominal transit time in terminal airspace giving them less opportunity to absorb delay with speed adjustments. This makes the Center's ability to deliver flows properly spaced for compression all the more important.

When distance separation requirements are given, the ORC arrival scheduler model converts the distances into time slots using the airspeeds published in STARs. The published airspeeds are $280 \mathrm{kn}$ or $250 \mathrm{kn}$ at the meter fix for all long and short side STARs respectively ${ }^{\mathrm{b}}$. These speeds equate to 77.1-100.8 sec time slots between arrivals at the meter fix to accommodate 6-7 NM separation with no wind. The same time slots would provide 8.1-9.8 NM separation in a $100 \mathrm{kn}$ tail wind, which is consistent with the 8-10 NM separation preferred by northwest corner controllers. Therefore, the model's use of published airspeeds to calculate static time separation requirements accommodates the controller's desire to modify distance separation to match wind conditions.

The arrival scheduler accommodates Miles-In-Trail (MIT) restrictions placed on a meter fix by replacing the nominal 6 or 7 NM separation with MIT distance. The MIT distance is converted to time in the same manner as the nominal separation, using published airspeeds. As with the nominal separations, for any given MIT, the time separation requirement is held constant such that the actual distance separation fluctuates with wind.

Runway distance separations are specified in TBFM adaptation by weight class pair of ahead/behind aircraft. The scheduler distance to time slot conversion assumes a landing speed of $145 \mathrm{kn}$. The time slots corresponding to the most common ahead/behind aircraft distance separations of $2.5 \mathrm{~nm}$ in 2-runway configurations and $3.2 \mathrm{~nm}$ in 3-runway configurations are 62.1 and $79.5 \mathrm{sec}$ respectively.

Table 1 summarizes the nominal distance separation to time slot conversions for the ORC arrival scheduler model. Note that although only the most common runway separation (Large Jet behind a Large Jet) is shown in the table, the scheduler uses the entire runway distance separation matrix containing all weight class combinations.

Table 1. Distance separation to time slot conversion.

\begin{tabular}{llll}
\hline \hline meter fix separation (long side) & distance (NM) & speed (kn) & time slot (sec) \\
\hline NW meter fix & 7.0 & 280 & 90.9 \\
other meter fixes & 6.0 & 280 & 77.1 \\
\hline \hline meter fix separation (short side) & distance (NM) & speed (kn) & time slot (sec) \\
\hline NW meter fix & 7.0 & 250 & 100.8 \\
other meter fixes & 6.0 & 250 & 86.4 \\
\hline \hline common runway separation & distance (NM) & speed (kn) & time slot (sec) \\
\hline 2-runway configurations & 2.5 & 145 & 62.1 \\
3-runway configurations & 3.2 & 145 & 79.5
\end{tabular}

\section{IV.B.2. Transit Time Ranges}

The arrival scheduler computes the transit time from meter fix to runway along predefined nominal terminal routes published in TBFM adaptation. The transit time range constraint used by the arrival scheduler is the nominal transit time (calculated using nominal airspeed in no wind) plus the maximum amount of delay that can be absorbed with speed along the route, called a delay buffer. All residual delay is passed back to Center airspace as meter fix delay. Currently fielded TBFM at IAH uses a single configurable delay buffer parameter (set at $60 \mathrm{sec}$ ) for all terminal routes. Even though there is some variation in the amount of delay different terminal routes can absorb with speed, it was determined that the uniform 60 sec delay buffer was sufficient for the purposes of estimating scheduled meter fix delay 90 minutes in advance.

\footnotetext{
${ }^{\mathrm{b}}$ Long side routes must complete a base leg turn onto final approach, which lengthens the route allowing meter fix airspeeds feeding long side routes to be greater than for short side routes.
} 


\section{Proof-of-concept Experiment}

In fast-time simulation, the TMC shown in Fig. 2 is circumvented by immediately implementing all ORC suggested reroutes. When ORC predicts arrival route congestion, the reroutes in the resulting ORC solution are implemented by updating FACET flight plans. The next simulation time cycle begins with the FACET trajectory prediction using the updated routes one minute of simulation time later.

\section{V.A. Experiment Setup}

The proof-of-concept focuses on clear-weather arrival operations into IAH during a rush from the Northeast challenging enough to exercise and stress the ORC algorithm. The day Oct 22, 2014 was chosen to seed a traffic scenario. On that day, IAH had high traffic volume, low weather impacts and was dominated by the W3 runway configuration (West flow, 3 arrival runways) which has the greatest runway capacity. To eliminate the effects of mirrored metering as discussed in section IV.B, flight plans were altered as necessary such that all flights filed RNAV STARs utilizing one of the four major meter fixes, WHACK (Northeast), MPORT (Northwest), GMANN (Southwest) and LINKK (Southeast). Whereas this day may have resulted in a few ORC suggested reroutes, a more aggressive arrival demand was desired to stress the algorithm so that it's behavior could be analyzed. FAA operations experts created a more challenging rush from the Northeast by cloning 12 of the 151 original flights and placing them at peak times to increase the load at WHACK.

Figure 6 shows the scenario demand on the four IAH meter fixes in 15-minute increments. Between 11:30 and 12:00, the northeast rush hits the northeast corner-post meter fix, WHACK. During this time, not only is the demand on WHACK high, but it is more than twice the demand on any other single meter fix.

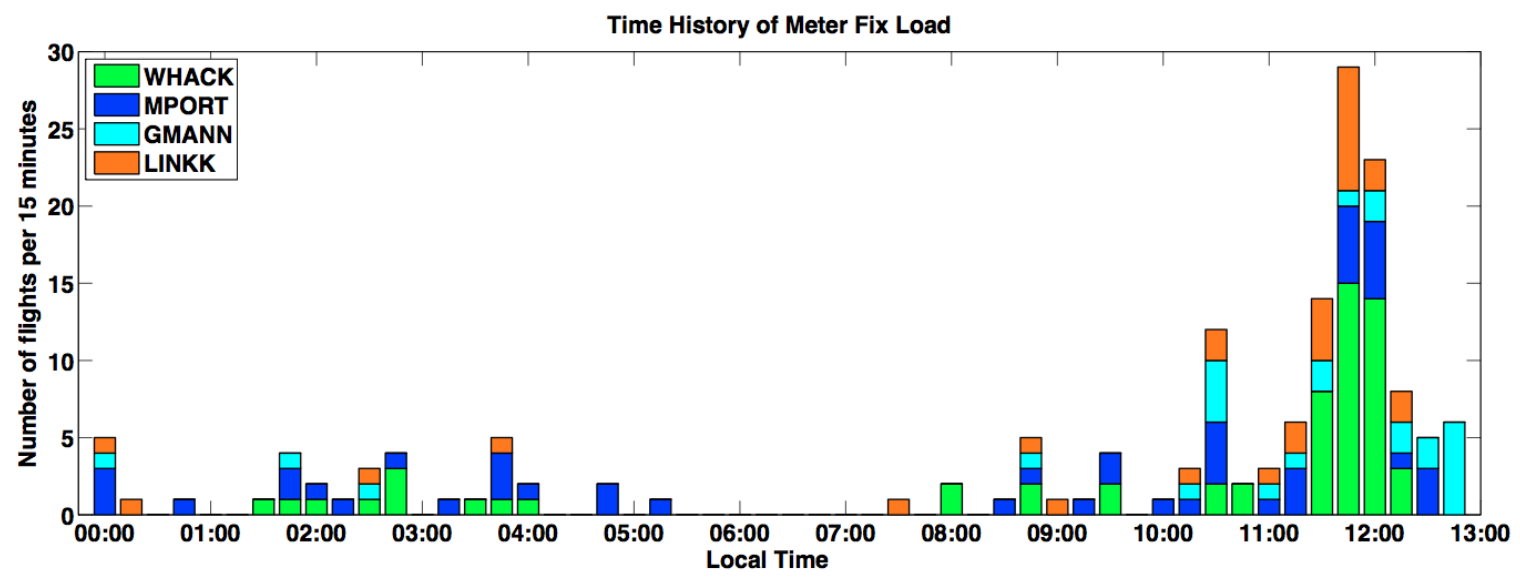

Figure 6. IAH arrival traffic scenario demand meter fix load per quarter hour.

Figure 7 shows a map view of IAH arrival flight positions (green triangles) at 11:00, approximately 1 hour before the full force of the Northeast rush will hit WHACK. The dashed circles are range rings emanating from IAH in increments of 100 miles. Orange triangles highlight flights that are estimated to have meter fix delay exceeding 7 minutes which ORC would detect as predicted arrival route congestion.

This scenario was simulated with and without automatic implementation of ORC reroutes to demonstrate the ORC reroute selection process and its effects on arrival operations. The ORC simulations used a 90minute planning horizon, meaning at any time during the simulation, ORC had knowledge of only flights with runway ETAs within 90 minutes.

\section{V.B. Results}

This section analyzes the ORC reroute solutions and compares arrival operations between the baseline and ORC simulations. 


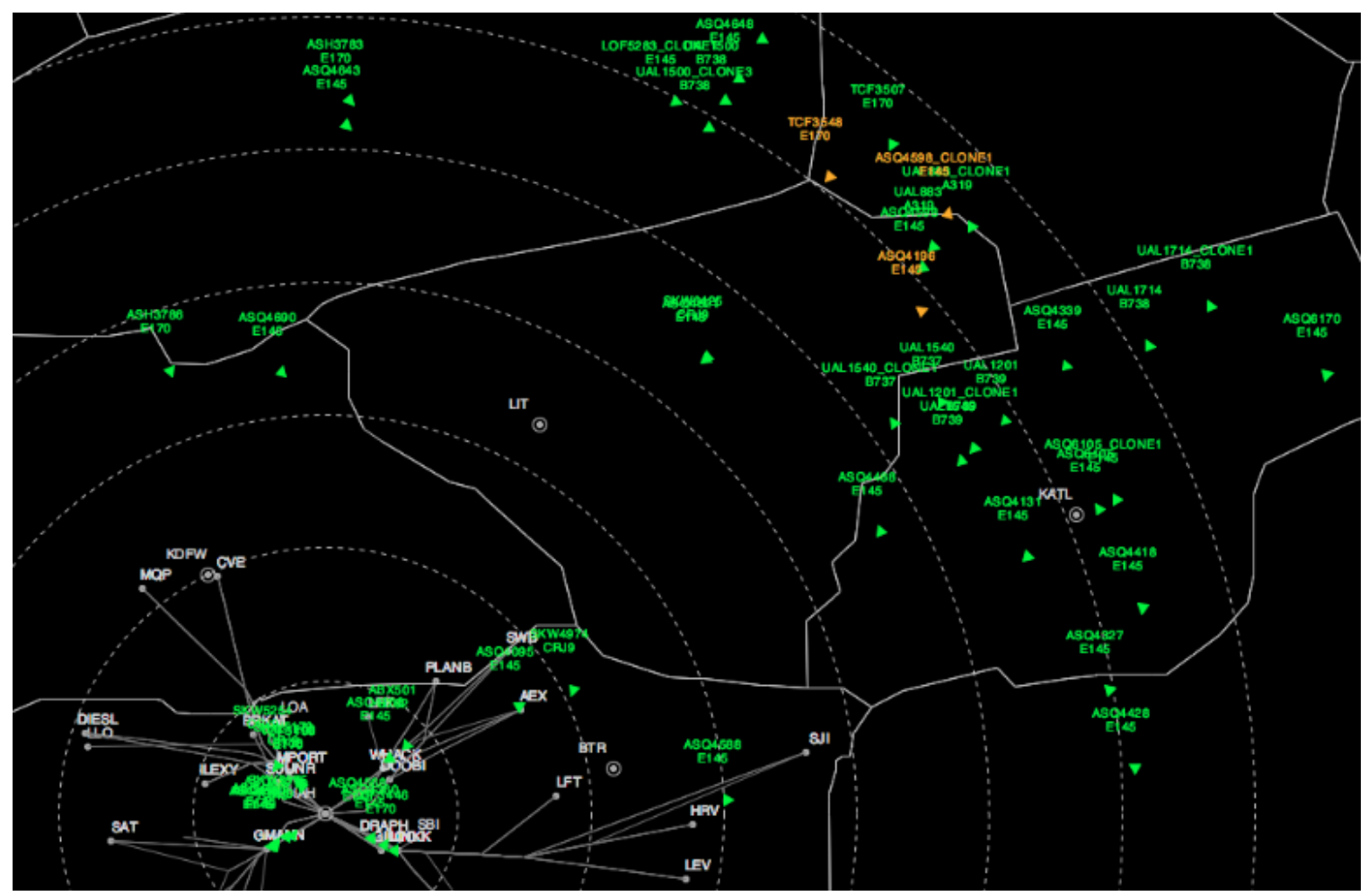

Figure 7. Map view of traffic scenario arrival rush from the Northeast.

\section{V.B.1. ORC Reroute Solutions}

Figure 8 shows the number of flights rerouted after each ORC action. Notice how eight ORC actions occurred in rapid succession over the span of roughly 15 minutes with usually no more than one reroute implemented per action. This is because ORC is limited to considering only flights within the planning horizon passing reroute availability filters. Within a minute of implementing an ORC solution, new flights may enter the ORC planning horizon with excess meter fix delay.

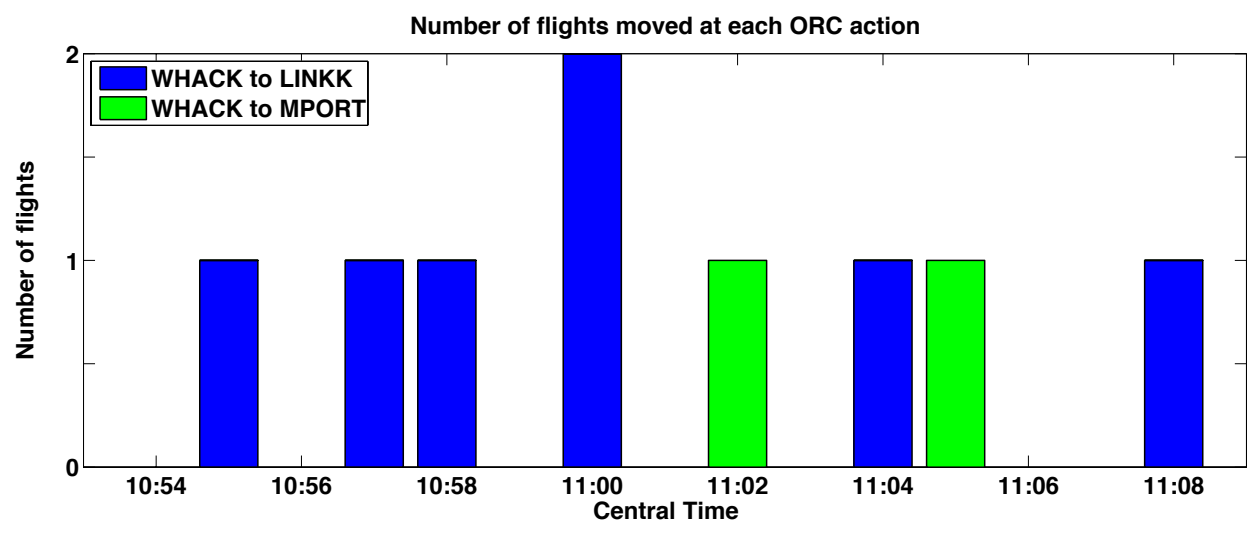

Figure 8. Number of flights ORC selected to reroute to alternate meter fixes.

Another reason for ORC triggering shortly after implementing a solution is that the previous solution may have only been a partial solution that did not completely remove the excess meter fix delay. For each ORC action, Figure 9 shows the scheduling cost (maximum excess meter fix delay) of the original routing and the ORC selected reroutes. At time 10:55 the excess delay just exceeds the 7-minute threshold and ORC finds a complete solution by moving a single flight South from WHACK to LINKK (see Figure 8 at 10:55). However, all successive ORC solutions are only able to partially solve the problem by reducing the 
scheduling cost, but not to zero.

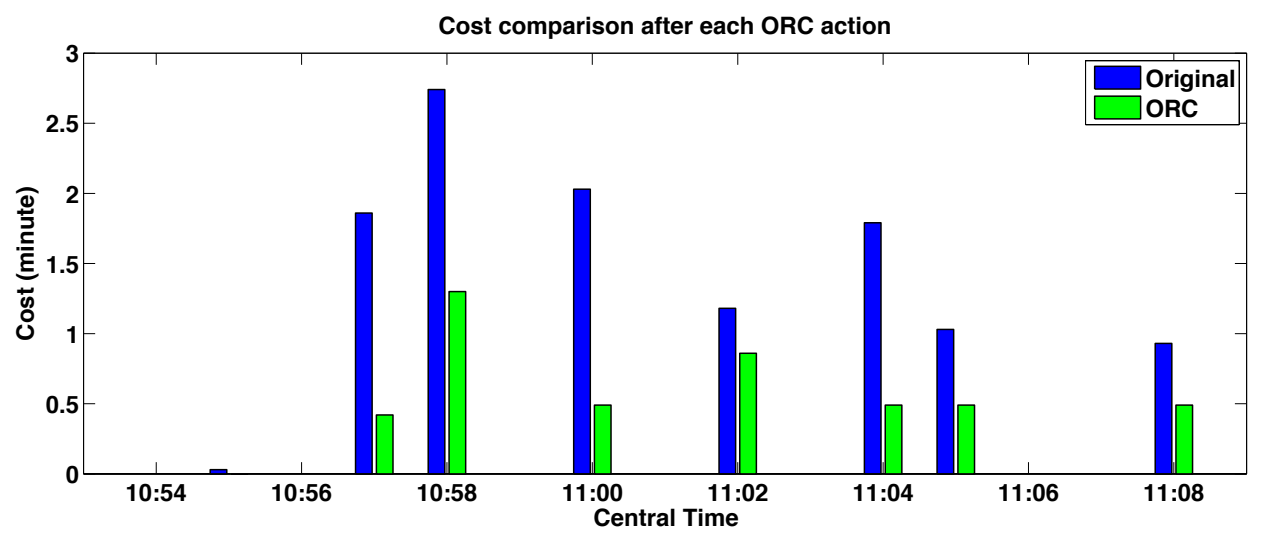

Figure 9. Comparison of scheduling cost before and after each ORC action.

One reason ORC may not find a complete solution may be the geographical filtering constraint excluding flights within 5 minutes from crossing a Center boundary for reroute consideration. Figure 7 shows how many flights entering the 90-minute planning horizon around the 700-mile range ring that could have been useful reroute candidates (flights highlighted orange and their followers) are just outside the Memphis Center boundary. Another reason for partial solutions may be that the airport runways were saturated by the demand resulting in excess meter fix delays at LINKK and MPORT when flights were offloaded to these meter fixes from WHACK.

Figure 10 shows the cumulative difference in runway STAs between the original routing and ORC solution. These differences correspond to the flights rerouted to alternate meter fixes and any succeeding flights within the planning horizon who's STA to their original meter fix changed as a result of moving the rerouted flights.

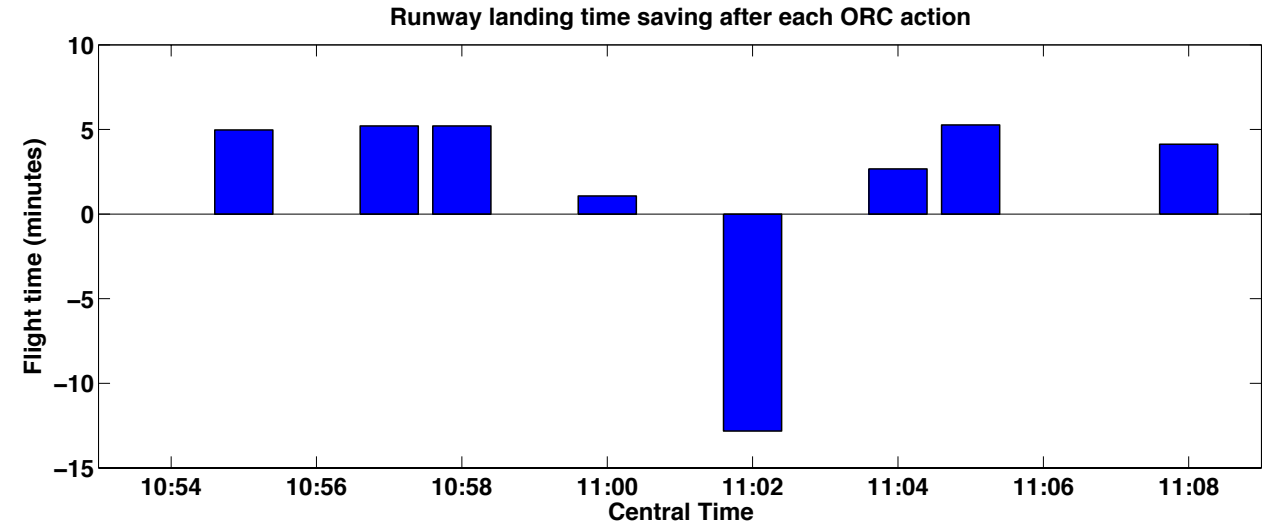

Figure 10. Difference in cumulative runway STA after each ORC action.

Notice how in most cases the ORC solution saved time. However, at 11:02 the ORC solution to move a single flight from WHACK to MPORT (see Figure 8 at 11:02) resulted in more time lost for the rerouted flight than saved by advancing the runway schedule of successive flights. ORC chose this solution because the maximum excess meter fix delay was reduced (see Figure 9 at 11:02). A traffic manager might have ignored this recommendation.

\section{V.B.2. ORC vs. Baseline}

The analyses presented in this section compare arrival operations between simulations with and without the automatic implementation of ORC reroutes. Whereas the previous analyses of ORC reroute solutions focused on the subset of flights within the ORC planning horizon at the time ORC selected each solution, the results in this section present a more global picture of the effect of ORC reroutes on arrival operations.

Figure 11 shows the airport demand and schedule load time histories for the simulations with original (baseline) routing and ORC reroutes. Each column represents the total number of ETAs or STAs for all 
runways within 15-minute bins The plot focuses on the time range between 11:00 and 14:00 where the effects of the Northeast arrival rush and differences between original and ORC routing can be seen.

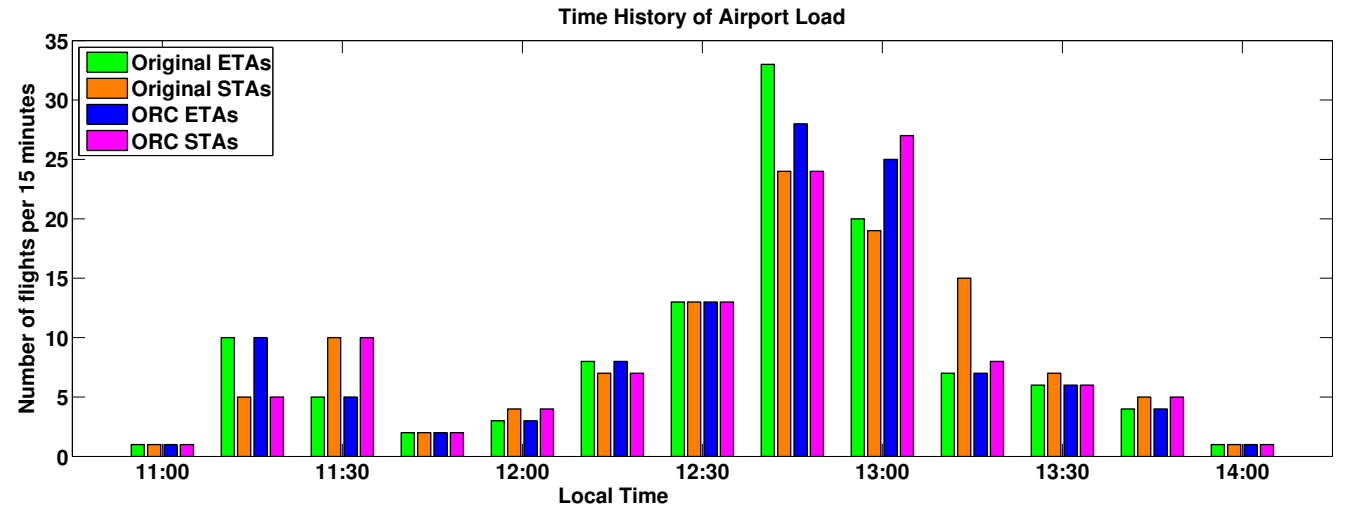

Figure 11. Airport demand and schedule loading per 15 minutes.

Through 12:30, original and ORC airport loading is identical although the effects of scheduling can be seen in the differences between ETA and STA loading for both. For example, 5 flights with ETAs in the 11:15 bin are shifted to STAs in the 11:30 bin in both the original and ORC simulations. The differences between original and ORC airport loading begin in the 12:45 bin where the same number of flights are scheduled to land but ORC reroutes shift several ETAs to the next bin. Consequently, these ORC reroutes allowed more flights to be scheduled to land in the 13:00 bin, increasing airport capacity utilization. ORC rerouting allowed the airport to largely recover from the airport demand/capacity imbalance in the 13:15 bin, when the original routing STA count is double the ETA count and the airport is still recovering from delays applied to ETAs from previous bins.

Figure 12 shows cumulative delays for each simulation (with and without ORC reroutes) segregated by the source of the delay. The Enroute and TRACON path delays are differences in nominal transit time through each domain between original and final routing. Enroute path delay is the difference in meter fix ETA between each flight's original route and reroute to an alternate meter fix. TRACON path delay is a similar difference in runway ETA minus the enroute path delay. Schedule delay is the difference between the reroute ETA (original route if not rerouted) and final STA at the runway.

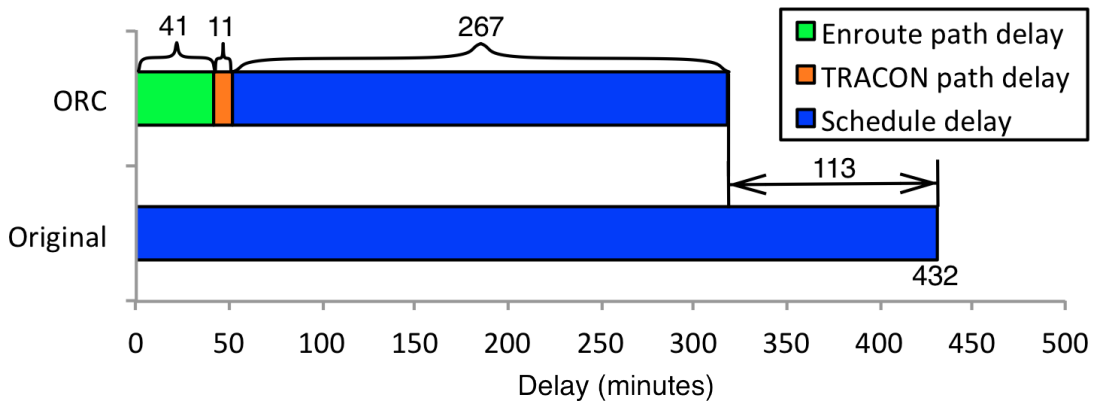

Figure 12. Cumulative delay segregated by source.

The original routing simulation without ORC did not have any path delay because there were no reroutes. ORC reroutes increased nominal flight time enroute by 41 minutes and in the TRACON by 11 minutes. However, this caused much less scheduled delay to be applied. The ORC reroutes reduced total delay by 113 minutes or $26 \%$. Note that this reduction in total delay is far greater than what can be seen within the ORC planning horizon in Figure 10. All the total delay savings (i.e. landing time difference) from Figure 10 sum to only 15 minutes. However, these planning horizon limited calculations do not account for the fact that each instance of delay or flight time savings may propagate back to all flights beyond the planning horizon. 


\section{Conclusion}

This proof-of-concept analysis of ORC applied to a northeast arrival rush into IAH demonstrates how strategically offloading flights from congested arrival routes can potentially provide substantial benefits to arrival operations. The ORC algorithm actively seeks to minimize excess meter fix delay which has the potential to reduce traffic manager workload and the use of other traffic management initiatives upstream such as miles-in-trail and ground delays. It also has the additional potential benefit of maximizing airport capacity utilization and reducing arrival scheduling delays. Future research is needed to explore whether the use of other scheduling cost functions driving the ORC solutions, or incorporating reroute cost into the selection of partial solutions, can further increase these additional potential benefits.

This phase of ORC assumed low weather impact conditions. Following phases will incorporate strategic weather avoidance routing to make this a valuable concept in all weather conditions.

\section{Acknowledgments}

The authors would like to recognize members of the original ORC concept development team, Philip Basset and Lisa Smith of FAA, Kenneth Hailston, Robert Giacomazzo, Carl Bernten, and Joel Hiks of Booz Allen Hamilton, and Mike Babbidge of Delta Airlines.

\section{References} 2013.

${ }^{1}$ Federal Aviation Administration. Optimized Route Capability - Preliminary functional analysis, Washington, D.C., July

${ }^{2}$ Federal Aviation Administration. Optimized Route Capability - Preliminary operational requirements, Washington, D.C., October 2013.

${ }^{3}$ Federal Aviation Administration. Optimized Route Capability - A Revised Concept Of Operations, Washington, D.C., September 2015.

${ }^{4}$ Bateman, H., C. Guensch, S. Heitin, and S. Kamine, Optimized Route Capability (ORC) Research Assessment, MITRE Technical Report MTR130600, McLean, VA, November 2013.

${ }^{5}$ Bilimoria, K., B. Sridhar, G. Chatterji, K. Sheth, and S. Grabbe, FACET: Future ATM Concepts Evaluation Tool, 3rd USA/Europe Air Traffic Management R\&D Seminar, Napoli, Italy, June 2000.

${ }^{6}$ Eurocontrol Experimental Centre, Base of Aircraft DATA (BADA) Aircraft Performance Modelling Report, EEC Technical/Scientific Report No. 2009-009, 2009.

${ }^{7}$ Meyn, L., A Closed-Form Solution to Multi-Point Scheduling Problems, AIAA Modeling and Simulation Technologies Conference, Toronto, Canada, August 2010. 